\title{
Synthesis and characterization of magnetically separable visible-light photocatalyst for removal of methyl orange
}

\author{
Rahmatollah Rahimi*, Parnian Kharazi, Mahboubeh Rabbani \\ Department of Chemistry, Iran University of Science and Technology, Tehran \\ 16846-13114, Iran
}

Rahimi_rah@iust.ac.ir

\begin{abstract}
Zinc ferrite-polythiophene nanocomposite was successfully prepared by in situ oxidative polymerization of thiophene monomers in presence of zinc ferrite. This composite were characterized by different techniques and photocatalytic-adsorption studies were carried out for removal of methyl orange (MO) from aqueous media. The result shows that the synthesized products induced a high photocatalytic-adsorption activity for removal of the MO dye. The synthesized sample can be separated easily by an external magnet and reused twice more, and its activity was virtually unchanged, although a slight decrease was observed after each run.
\end{abstract}

Keywords: Zinc ferrite, Polythiophene, Photocatalyst, Absorption.

\section{Introduction}

The presence of dyes in wastewater has become a significant source of water pollution, due to textile, paper and printing industries [1]. The removal of dyes in an economic way remains an 
important issue for researchers and environmentalists [2]. Photocatalytic elimination is an effective way to eliminate these pollutants dye [3]. The separation and recycling of the photocatalysts are still a serious problem. Fortunately, magnetic separation offers a convenient way to remove and recycle the magnetic composite materials properly by employing an external magnetic field $[4,5]$. For this purpose, recently combinations of conjugated polymers with inorganic magnetic materials has received great attention [6]. The unique physical and chemical properties of conducting polymers have made them significant attractive materials. Among these delocalized conjugated materials, polythiophenes polymers have been widely used because of their good thermal and chemical stability [7].

Effective photocatalytic oxidation of the organic pollutants strongly depends on the adsorption capacity of the photocatalyst. [8]. To enhance the adsorption process, coating of polythiophene onto zinc ferrite is considered a viable technique. In this study, zinc ferrite-polythiophene nanocomposite was synthesized to combine both advantages of zinc ferrite and polytiophene for removal of methyl orange (MO) from aqueous media.

\section{Experimental}

\subsection{Materials and Methods}

All of the chemicals used in this work were analytical grade reagents and used without further purification. Zinc nitrate, ferric nitrate, glycine, ammonium nitrate, thiophene, potassium permanganate, methyl orange and anhydrous acetonitrile were purchased from Merck company. Deionized water was used to prepare all solutions.

The samples were characterized by X-ray powder diffraction (XRD) using a Philips PW 1730 Xray diffractometer with $\mathrm{Cu} \mathrm{K \alpha}$ radiation. The morphologies of the samples were observed by an 
AIS2100 (Seron Technology) scanning electron microscopy (SEM). The FT-IR analyses were carried out on a Shimadzu FTIR-8400S spectrophotometer using a $\mathrm{KBr}$ pellet for sample preparation.

\subsection{Preparation of polythiophene coated on zinc ferrite nanoparticles}

For the preparation of this sample, zinc ferrite nanopatricles were first prepared by a combustion method. In this procedure, a mixed aqueous solutions prepared by dissolving required weights of zinc (II) and iron (III) nitrates, ammonium nitrate and glycine, as a fuel in $10 \mathrm{~mL}$ distilled water. The mixed precursors were concentrated in a porcelain crucible on a hot plate at $300{ }^{\circ} \mathrm{C}$. After the mixture reaches the point of spontaneous combustion, it began burning and a brown voluminous and fluffy $\mathrm{ZnFe}_{2} \mathrm{O}_{4}$ product remained in the container. The acquired substance was collected and then washed with distilled water and ethanol for several times and finally dried in an oven, at 80 ${ }^{\circ} \mathrm{C}$ for $5 \mathrm{~h}[9]$.

Then these magnetic nanoparticles were coated with polythiophene through polymerization of thiophene monomers on the surface of pre-synthesized $\mathrm{ZnFe}_{2} \mathrm{O}_{4}$ nanoparticles.

For this purpose, $0.5 \mathrm{~g}$ of magnetic nanoparticles were dispersed in $5 \mathrm{~mL}$ anhydrous acetonitrile and underwent ultrasound for $10 \mathrm{~min}$. Then, $0.75 \mathrm{~mL}$ thiophene monomers was added to the NPs suspension and stirred for $15 \mathrm{~min}$. Afterwards, $25 \mathrm{~mL}$ of $6 \mathrm{mM} \mathrm{KMnO}_{4}$ solution in anhydrous acetonitrile was added to the mixture drop by drop. After addition of $\mathrm{KMnO}_{4}$ solution, the mixture was stirred for $3 \mathrm{~h}$. Finally, obtained product was rinsed by deionized water and ethanol several times and dried at $70{ }^{\circ} \mathrm{C}$ for $5 \mathrm{~h}$ under vacuum [10].

\subsection{Photocatalysis procedure}


In a typical process, the catalytic reaction was carried out in a $100 \mathrm{~mL}$ photoreactor, which contain $50 \mathrm{~mL}$ of MO dye $(10 \mathrm{mg} / \mathrm{L})$ solution and $5 \mathrm{mg}$ of catalyst. $5 \mathrm{~W}$ LED lamp was used as the light sources. In the experiment, the reaction solution was mixed by magnet stirrer which was placed under light source. Before exposure to light the solution was stirred in the dark for $30 \mathrm{~min}$ to allow adsorption of methyl orange. Samples $(3 \mathrm{~mL})$ were collected during the absorption-photocatalytic reaction and $\mathrm{MO}$ solution were separated from by centrifugation. The concentration of $\mathrm{MO}$ was determined by measuring the absorbance amount at its maximum absorbance wavelength of MO using a double beam UV-Vis spectrophotometer.

\section{Results and Discussion}

\subsection{FT-IR spectroscopy of polythiophne coated on zinc ferrite nanoparticles}

FT-IR spectra of polythiophene coated on zinc ferrite nanoparticles are shown in Fig. 1. The strong and sharp absorption band at the range between $500-600 \mathrm{~cm}^{-1}$ is attributed to M-O bonds vibration as a typical band of spinel ferrite. The peaks corresponded to polythiophene are appeared between $1000-2000 \mathrm{~cm}^{-1}$. 


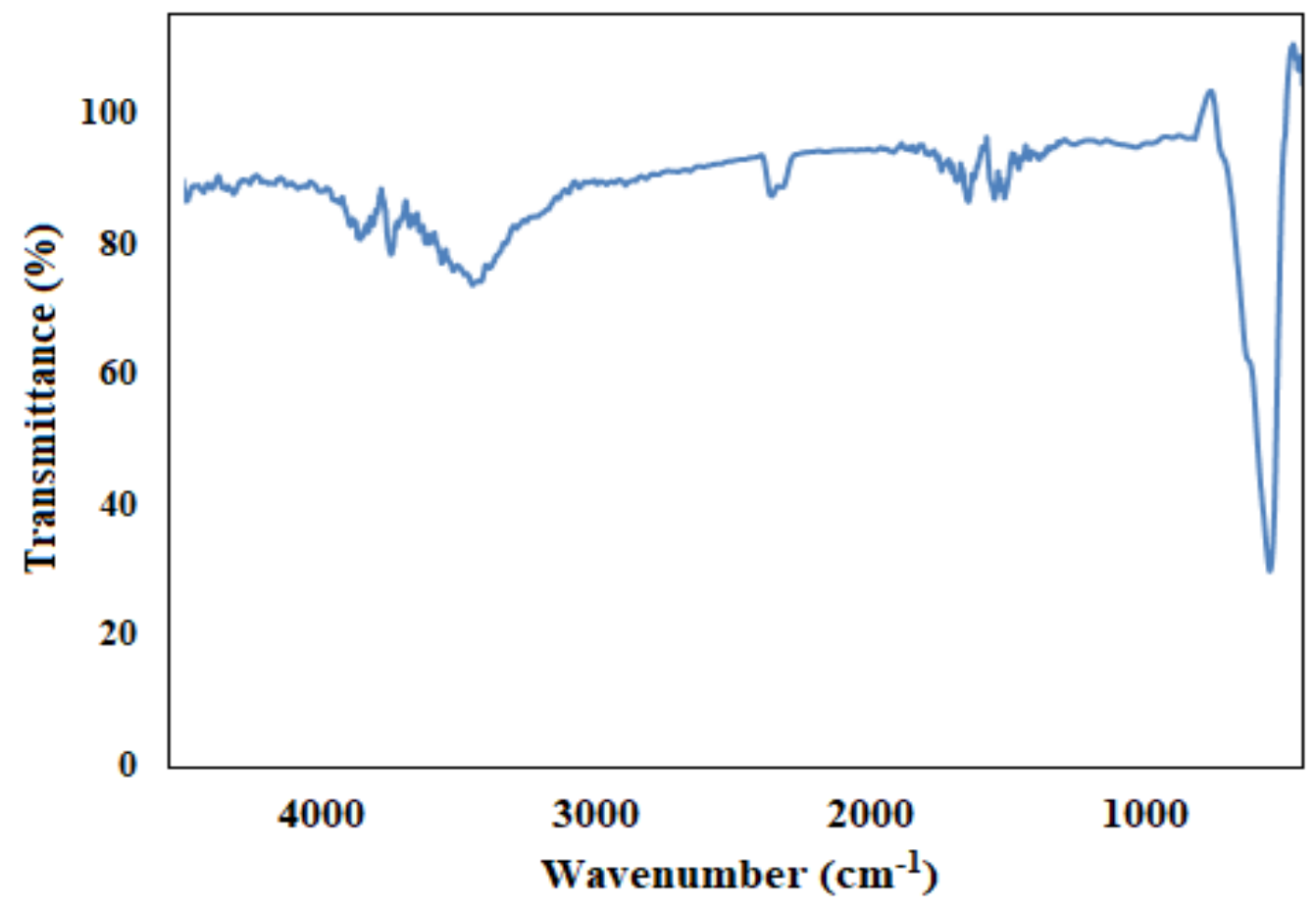

Fig. 1. The FT-IR spectrum of polythiophene coated on $\mathrm{ZnFe}_{2} \mathrm{O}_{4}$

\subsection{XRD diffraction pattern of polythiophene coated on zinc ferrite nanoparticles}

Fig. 2 illustrates XRD pattern of the nanocomposite. The X-ray diffraction pattern of the asprepared composite shows the expected peaks for pure inverse spinel structure of $\mathrm{ZnFe}_{2} \mathrm{O}_{4}$ and reveal that sample is single phase $\mathrm{ZnFe}_{2} \mathrm{O}_{4}$ and all diffraction peaks can be indexed to cubic spinel structure of zinc ferrite. 


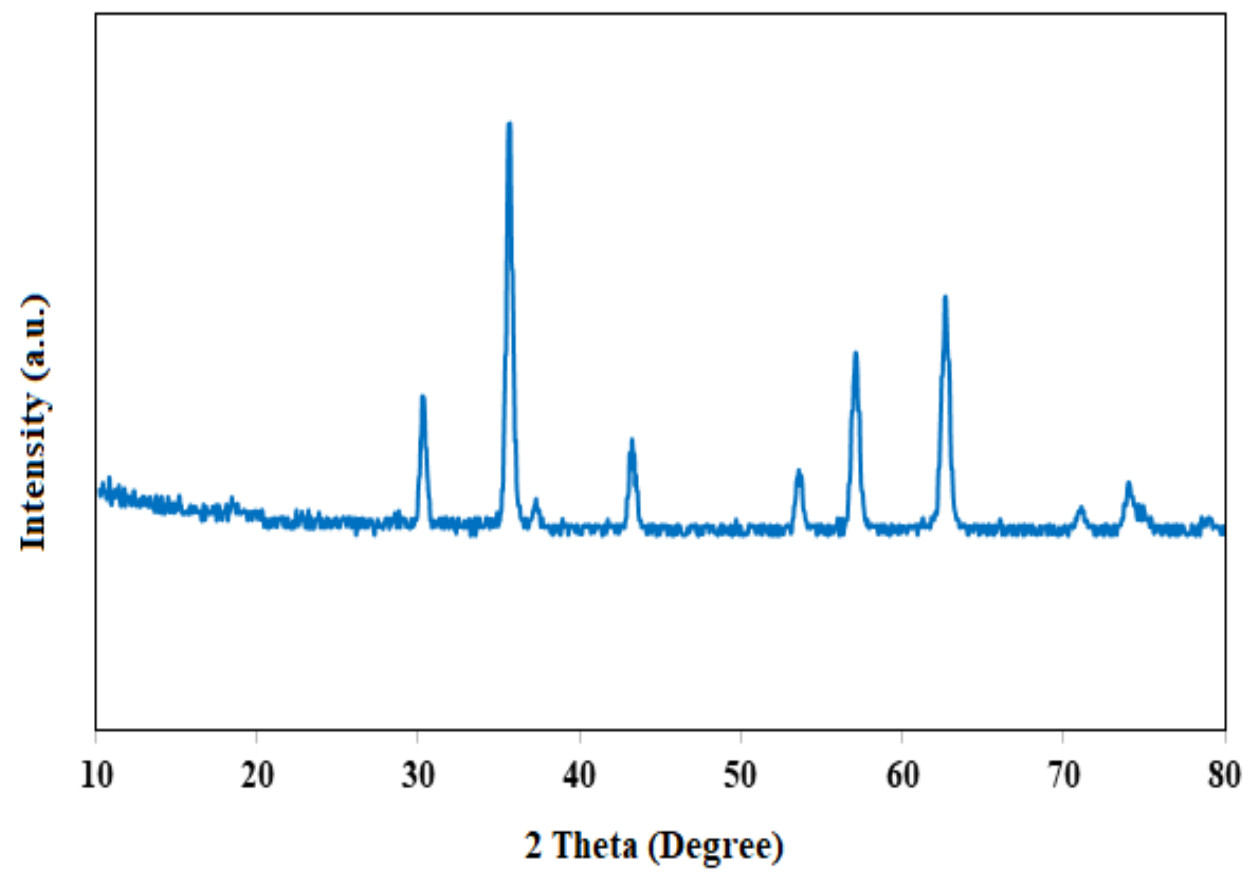

Fig. 2. The XRD pattern of polythiophene coated on $\mathrm{ZnFe}_{2} \mathrm{O}_{4}$

\subsection{Morphology of polythiophene coated on zinc ferrite nanoparticles}

The SEM micrograph of polythiophene coated on zinc ferrite is shown in Fig. 3. As shown in this figure, the SEM micrographs exhibit some agglomeration due to magnetic dipole interaction between ferrite particles.

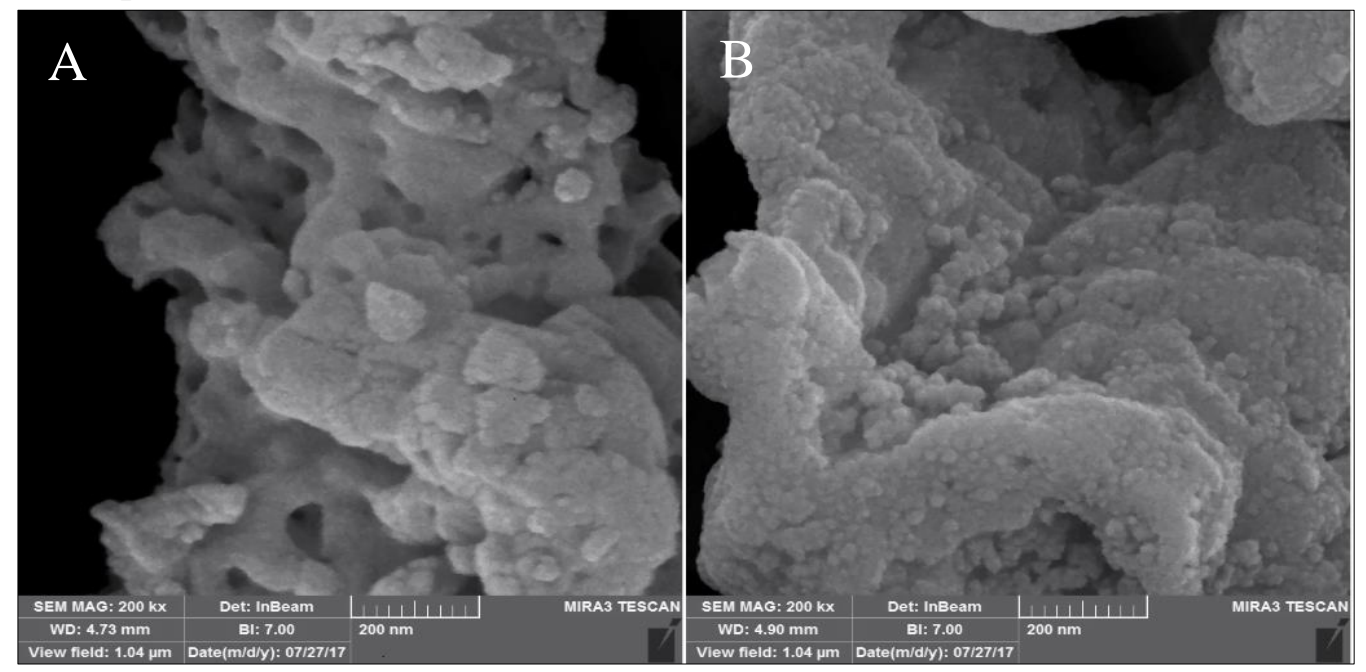

Fig. 3. SEM images of (A) zinc ferrite and (B) polythiophene coated on zinc ferrite. 


\subsection{EDX analysis of polythiophene coated on zinc ferrite nanoparticles}

The elemental analysis of polythiophene coated on zinc ferrite nanoparticles has been carried out using EDX techniques. Fig. 4 illustrate the presence of of oxygen, sulfur, zinc and iron obtained from EDX analysis, that verify coating of polythiophene on surface of ferrite nanoparticles.

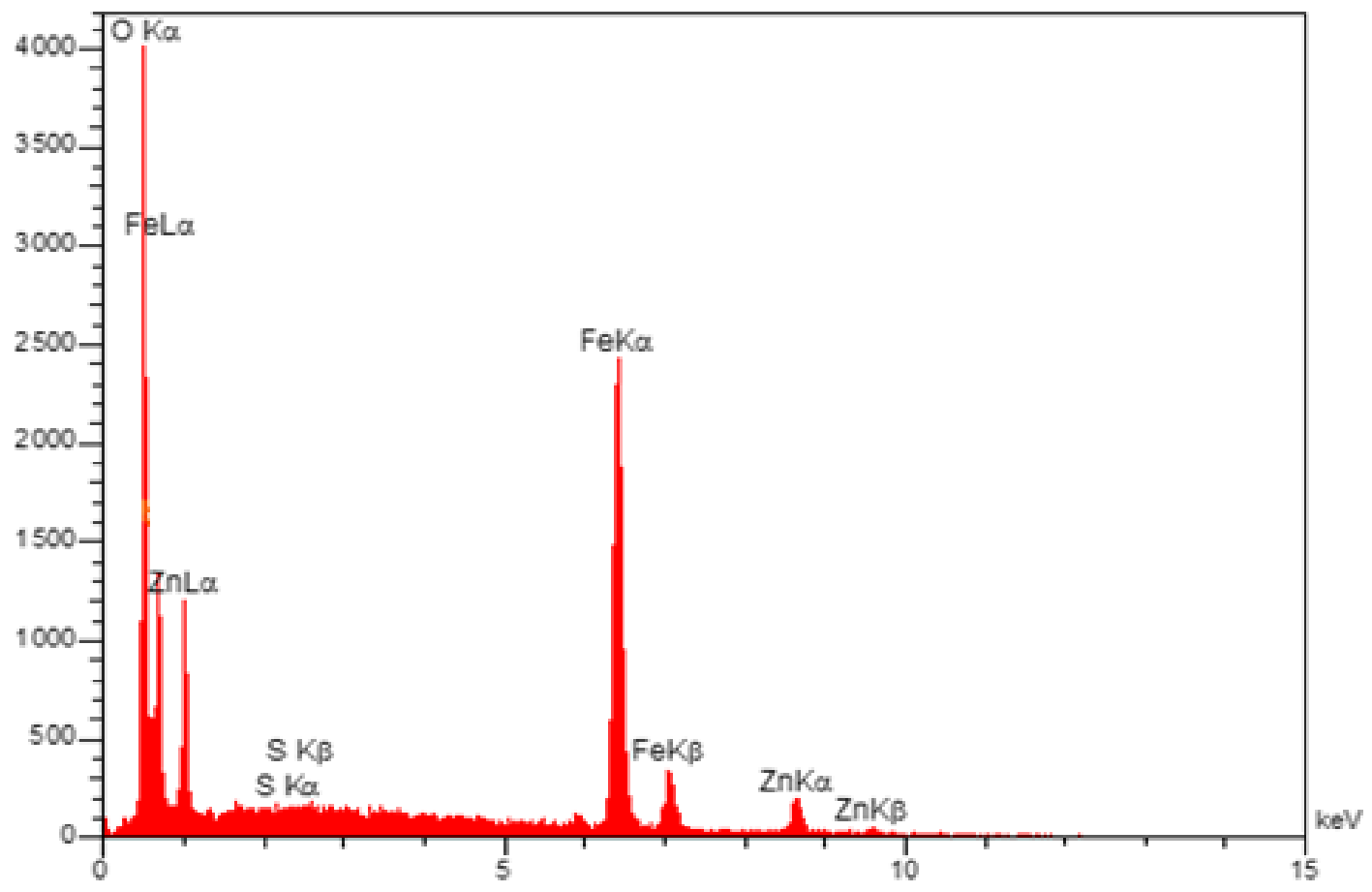

Fig. 4. EDX analysis of polythiophene coated on zinc ferrite

\subsection{Adsorption and photocatalytic degradation of methyl orange}

The adsorptions and photocatalytic activities of zinc ferrite and polythiophene coated on zinc ferrite photocatalysts was defined by measuring the removal of $\mathrm{MO}$ aqueous solutions in dark and under visible LED light irradiation (Fig. 5). The dark condition was tested to investigation of adsorbing properties of catalysts. The results show that removal efficiency of MO in presence of zinc ferrite and polythiophene coated on zinc ferrite was carried out by adsorbing rather than 
photocatalytic degrading. On the other hands, the presence of polythiophene significantly enhanced removal efficiency.

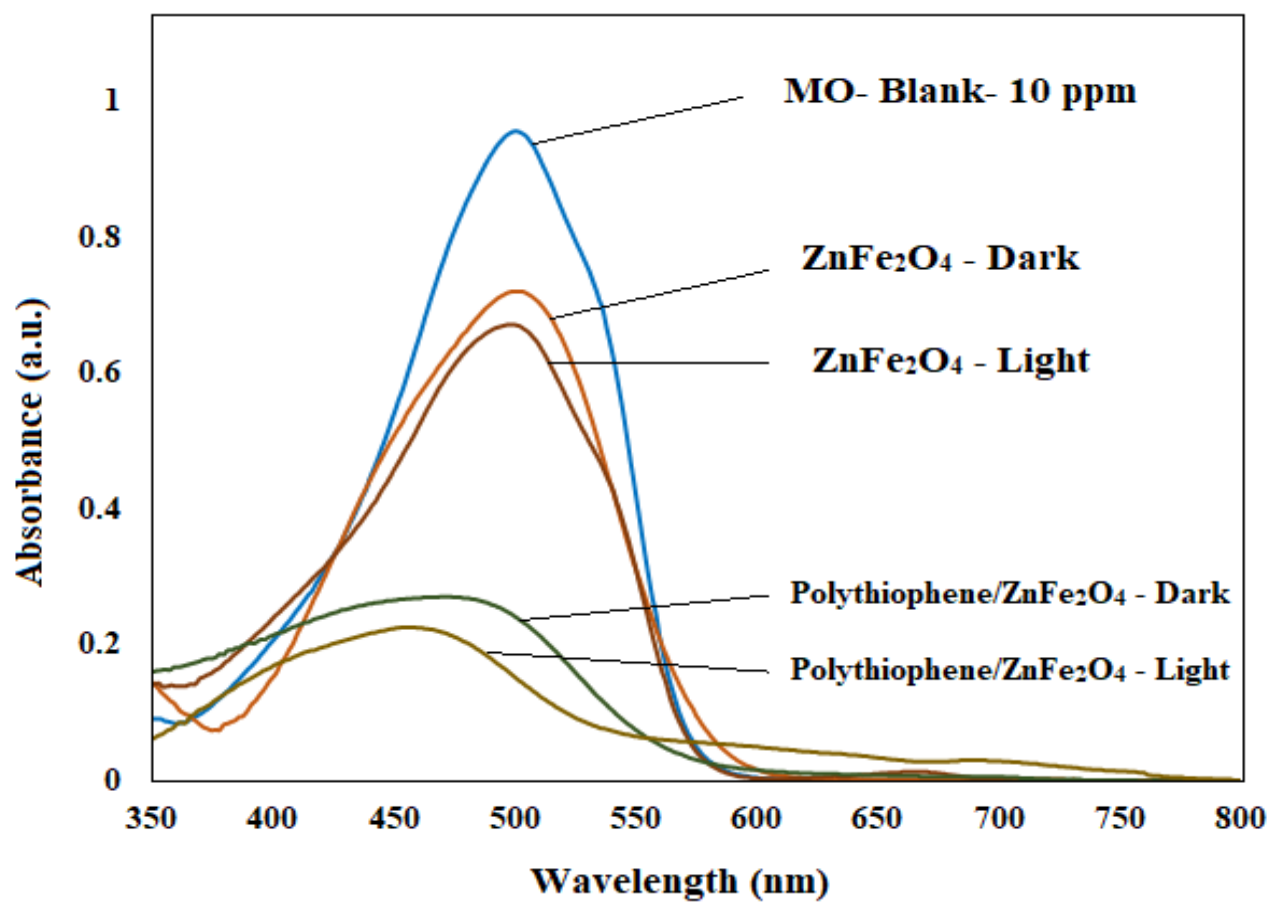

Fig. 5. The UV-Vis spectra for degrading of MO (10 ppm) in dark and under visible light irradiation

\section{Conclusions}

This research demonstrated successful synthesize of polythiophene coated on zinc ferrite via oxidative polymerization of thiophene in the presence of zinc ferrite using $\mathrm{KMnO}_{4}$ as the oxidant. The results indicated that the as-synthesized polythiophene coated on zinc ferrite nanocomposite exhibit excellent magnetic properties and can be taken for removal of methyl orange dye from wastewater. 


\section{References}

[1] A. A. Spagnoli, D. A. Giannakoudakis, S. Bashkova, Journal of Molecular Liquids 229 (2017) $465-471$.

[2] D. Pathania, Sh. Sharma, P. Singh, Arabian Journal of Chemistry 10 (2017) S1445-S1451.

[3] M. Rabbani, M. Heidari-Golafzani, R. Rahimi, Materials Chemistry and Physics (2016) 1-7.

[4] W. Qiu, Y. Zheng, K. A. Haralampides, Chemical Engineering Journal 125 (2007) 165-176.

[5] Z. Zhang, L. Xu, Ch. Liu, RSC Avances, 5 (2015) 79997-80004.

[6] K. Nam Kim, H. Jung, W. Lee, Journal of Photochemistry and Photobiology A: Chemistry 321 (2016) 257-265.

[7] M. Aydin, Z. Durmus, H. Kavas, B. Esat, H. Sozeri, A. Baykal, F. Yilmaz, M.S. Toprak, Polyhedron 30 (2011) 1120-1126.

[8] M. Rabbani, M. Haghverdi, M. Heidari-Golafzani, R. Rahimi, Marziye Javaheri Kachousangi, New Journal of Chemistry, 2017, 41, 8011-8015.

[9] N. M. Deraz, A. Alarifi, International Journal of Electrochemical Science, 7 (2012) 6501 6511.

[10] E. Tahmasebi, Y. Yamini, M. Moradi, A. Esrafili, Analytica Chimica Acta 770 (2013) 6874. 